Research Paper

\title{
Methods to preserve potentially toxigenic fungi
}

\author{
Lucas Costa Guimarães ${ }^{1}$, Ana Paula Fernandes ${ }^{2}$, Sara Maria Chalfoun ${ }^{3}$, \\ Luís Roberto Batista ${ }^{2}$ \\ ${ }^{1}$ Instituto de Ciências da Saúde, Universidade Paulista, Brasília, DF, Brazil. \\ ${ }^{2}$ Departamento de Ciência dos Alimentos, Universidade Federal de Lavras, Lavras, MG, Brazil. \\ ${ }^{3}$ Empresa de Pesquisas Agropecuária de Minas Gerais, Lavras, MG, Brazil.
}

Submitted: June 12, 2012; Approved: September 09, 2013.

\begin{abstract}
Microorganisms are a source of many high-value compounds which are useful to every living being, such as humans, plants and animals. Since the process of isolating and improving a microorganism can be lengthy and expensive, preserving the obtained characteristic is of paramount importance, so the process does not need to be repeated. Fungi are eukaryotic, achlorophyllous, heterotrophic organisms, usually filamentous, absorb their food, can be either macro or microscopic, propagate themselves by means of spores and store glycogen as a source of storage. Fungi, while infesting food, may produce toxic substances such as mycotoxins. The great genetic diversity of the Kingdom Fungi renders the preservation of fungal cultures for many years relevant. Several international reference mycological culture collections are maintained in many countries. The methodologies that are most fit for preserving microorganisms for extended periods are based on lowering the metabolism until it reaches a stage of artificial dormancy. The goal of this study was to analyze three methods for potentially toxigenic fungal conservation (Castellani's, continuous subculture and lyophilization) and to identify the best among them.
\end{abstract}

Key words: mycotoxins, toxigenic fungi, methods for fungal conservation.

\section{Introduction}

Microorganisms are sources of many high value compounds that are useful to all living beings. Some of the most important products that use microorganisms in their production are vitamins, antibiotics, alcohol, enzymes, biosurfactants, medicines etc. (Cameotra, 2007).

The international community considers the XXI century the Biotechnology era, where, mycelial fungi are considered large biotechnology producers. In recent decades, these organisms have been biologically employed for the obtaining a series of active substances that are used in agriculture, the food industry and especially in medicine. (Feofilova et al., 2009).

The isolation and improvement of a microorganism are long and expensive processes, thus it is essential to preserve the characteristic obtained so as not to need to repeat those procedures once again. The choice of a preservation technique for specific microorganism depends on the char- acteristics of the method, maintenance costs, importance of the collection and equipment availability, among other factors.

The preservation of fungal cultures is an essential element of systematics and biodiversity studies, because the fungi are a widely diverse group and for that various cultivation and preservation methods are necessary to guarantee the viability and morphological, physiological and genetic integrity of the cultures over time. The cost and convenience of each method, however, should also be considered. (Nakasone et al., 2004).

Knowing how to preserve culture is to have simple and efficient techniques for such, it is of the most conspicuous importance in any laboratory where research activities are developed. (Romeiro, 1996).

The importance of culture preservation arises from the need to have the organism or specimen available at any time, for experimental ends, either for routine works or to

Send correspondence to L.C. Guimarães. Instituto de Ciências da Saúde, Universidade Paulista, Campus Brasília, Brasília, DF, Brazil. E-mail: luguima18@hotmail.com. 
meet requests from other researchers, for didactic ends, for comparative studies, etc. (Samson, et al., 2004).

Seeking to obtain the best method of microorganism preservation, the present work proposes to apply three preservation methods of potentially toxigenic fungi isolated from foods and to identify the best among them.

\section{Methodology}

\section{Collection of food samples}

The samples were obtained from the retail market of the city of Lavras - MG, including unprocessed foods with deterioration signs (pear, potato, grape, Brazil nut, peanut, wheat) and foods still processed within the expiration period (canned corn, linseed, raisins and peanuts) as observed in Table 1. The samples obtained were taken to the EPAMIG/URESM Laboratory at the Federal University of Lavras where the microbiological analyses were conducted.

\section{Microbiological analysis}

The analyses of the samples were carried out through direct plating, which consisted of removal of fragments of the injured area of the food or the sample in an integral manner and aseptically transferring them to Petri dishes with PDA medium (Potato Dextrose Agar).

After plating, the dishes were maintained in BOD with temperature of $25{ }^{\circ} \mathrm{C}$ and a photoperiod of $12 \mathrm{~h}$, for 5 days. After that period, the microorganisms were purified, characterized, identified and stored.

\section{Characterization}

The characterization was conducted through macroscopic and microscopic characteristics. The macroscopic characteristics appraised were coloration and colony diameter, sclerotia presence or absence and coloration, coloration of the underside of the colony in different media, among others.

Table 1 - Fungi obtained and origin.

\begin{tabular}{lc}
\hline Fungi & Origin \\
\hline Aspergillus carbonarius & Coffee \\
Aspergillus niger & Grape \\
Aspergillus niger Agregados & Coffee \\
Aspergillus ochraceus & Potato \\
Aspergillus parasiticus & Brazil-nut \\
Aspergillus versicolor & Coffee \\
Aspergillus flavus & Brazil-nut \\
Penicillium aurantiogriseum & Coffee \\
Penicillium chrysogenum & Peanut \\
Penicillium citrinum & Wheat \\
Penicillium commune & Mycology collection EPAMIG/CTSM \\
Penicillium expansum & Coffee \\
\hline
\end{tabular}

Among the microscopic characteristics studied were the length of the conidiophores, forms and size of the conidia, texture of the conidia and conidiophores.

The identification of the species of the gerera Aspergillus was according to Klich (Klich, 2002) and Christensen (Christensen, 1982); the genus Penicillium according to Pitt (Pitt, 1988), and Samson et al. (Samson, et al., 2004), where we only selected the fungi with toxigenic potential (Table 2).

\section{Preservation methods}

\section{Lyophilization}

The samples of the fungi were frozen in duplicate at $-80{ }^{\circ} \mathrm{C}$ in an ultra-freezer, after the freezing the samples were taken to the Liotop, I model L101 lyophilizer, where the initial temperature was $-50 \pm 2{ }^{\circ} \mathrm{C}$. With the temperature stabilized, the vacuum was initiated with a pressure around $650 \mu \mathrm{Hg}$, after $48 \mathrm{~h}$ the lyophilization concluded with pressure around $150 \mu \mathrm{Hg}$.

Every two months the lyophilized fragments were isolated and put in 2\% Malt Agar (MA) medium for the viability test, and the fungi revealed as toxigenic were also placed in specific medium for the toxigenic potential test.

\section{Sterile distilled water}

The inoculation of glass flasks containing sterilized distilled water with a small portion of the culture medium (approximately $5 \mathrm{~mm} \times 10 \mathrm{~mm}$ ) with the fungus to preserve, was conducted. The flasks employed were the same used for the antibiotic, with a $6 \mathrm{~mL}$ capacity, filled with 4 $\mathrm{mL}$ of distilled water, sealed with their own rubber stopper and autoclaved at $121^{\circ}$ under $1 \mathrm{~atm}$ for $30 \mathrm{~min}$. After the autoclaving the transference of the fungi to the flasks with

Table 2 - Fungi and mycotoxins potentially produced.

\begin{tabular}{lc}
\hline Fungi & Principal mycotoxins \\
\hline Aspergillus carbonarius & Ochratoxin A \\
Aspergillus niger & Ochratoxin A \\
Aspergillus niger Agregados & Ochratoxin A \\
Aspergillus ochraceus & Ochratoxin A \\
Aspergillus parasiticus & Aflatoxin B1, B2, G1, G2 \\
Aspergillus versicolor & Esterigmatocistin \\
Aspergillus flavus & Aflatoxin B1, B2 \\
Penicillium aurantiogriseum & Auranthine, penicillic acid \\
Penicillium chrysogenum & Roquefortine c C \\
Penicillium citrinum & Citrinin \\
Penicillium commune & Cyclopaldic acid \\
Penicillium expansum & Patulin \\
\hline
\end{tabular}

Source: Illustrated Manual on Identification of Some Seed-borne Aspergilli, Fusaria, Pencillia and Their Mycotoxins. 
water in an aseptic chamber was carried out in duplicate. The flask stopper was removed and pieces of culture medium containing fungal mycelium were transferred into the flasks.

Every two months the fragments were isolated and placed in $2 \%$ MA medium for the viability test, and the fungi revealed as toxigenic were also placed in a medium specific for the toxigenic potential test.

\section{Continuous transference}

The transference of the fungi was carried out in duplicate. The fungi were placed in petri plates with $2 \%$ MA medium sealed with parafilm, the cultures were stored in a in refrigerator at a temperature from 4 to $8^{\circ} \mathrm{C}$ according to the literature, and every two months the fungi were transfered to other plates with $2 \%$ MA medium for the viability test, and those toxigenic were also placed in a medium specific for the toxigenic potential test.

\section{Results}

\section{Fungal isolate viability test}

In Table 3 the fungal isolates can be evaluated at time 0 , that is, before they were submitted to the preservation tests. They were found viable and pure.

Through the data shown in Table 4 the results of the viability tests of the isolates can be seen at times 1,2,3 and 4 corresponding to 2, 4, 6 and 8 months of preservation by the continuous subculture, Castellani and lyophilization methods, being represented by the + sign as viableand by the - sign as unviable.

According to the results obtained and represented by Table 4 one can observe that the isolates presented viability under the three preservation methods.

In relation to the methods used in the present work, the Castellani method is indicated as the most advantageous among the three methods used, therefore besides

Table 3 - Represents the viability of potentially toxigenic fungal isolates at time 0 .

\begin{tabular}{|c|c|}
\hline Fungi & Viability \\
\hline Aspergillus carbonarius & + \\
\hline Aspergillus niger & + \\
\hline Aspergillus niger Agregados & + \\
\hline Aspergillus ochraceus & + \\
\hline Aspergillus parasiticus & + \\
\hline Aspergillus versicolor & + \\
\hline Aspergillus flavus & + \\
\hline Penicillium aurantiogriseum & + \\
\hline Penicillium chrysogenum & + \\
\hline Penicillium citrinum & + \\
\hline Penicillium commune & + \\
\hline Penicillium expansum & + \\
\hline
\end{tabular}

having a high viability rate and preserving the characteristics of the isolates.

It was also observed that there weren't any changes to the macroscopic and morphological characteristics of the tested fungi.

\section{Discussion}

The fungi, unlike most of the bacteria, are beings with a slow growth rate in culture media. On the other hand, many times there is contamination by bacteria or other fungi, which harms the preservation of the colonies. The existent techniques for the mycology collection maintenance are difficult, costly and frequently inefficient. The development of new ways of fungal preservation of for prolonged periods becomes necessary. (Samson et al., 2004).

In a study using 26 fungi strains preserved by the Castellani method for 2 years the viability of $100 \%$ of the strains was observed, with no alterations in the macroscopic and morphological characteristics, demonstrating the efficiency of that method in the preservation of fungal culture viability of (e Bueno Gallardo, 1998).

Evaluating the results obtained we observed that they are in agreement with the affirmative of Cavalcanti (Feofilova et al., 2009). who obtained high viability in a study on the preservation by the Castellani, continuous subculture and lyophilization methods, also affirming the Castellani method as viable in the preservation of dimorphic fungi and the lyophilization method is shown more efficient in the preservation of yeasts, presenting of viability rate of $100 \%$ in the conducted study.

Previous studies comparing the viability of fungal cultures preserved by the Castellani and lyophilization methods suggest that the Castellani method (distilled water) is more advantageous to maintain, in laboratory, different gerera and species of fungi, even comparing the two methods(e Qiangqiang Jiajun, 1998) in another study comparing the viability of seventy eight isolates after twelve years, using the lyophilization and Castellani methods in the their preservation obtaining $89.7 \%$ of the isolates as viable preserved by the Castellani method and $87.2 \%$ of the isolates preserved by the lyophilization method. (Romeiro, 1996).

A study, where the use of the Castellani method and continuous subculture was compared in the preservation of hundred and eleven strains of different species of microorganisms during a seven-year the period, resulted in (71.2\%) viability in the strains preserved by the Castellani method and $(77.5 \%)$ viability in the strains preserved through continuous subculture.(Samson et al., 2004).

\section{Conclusion}

The lyophilization methods, Castellani and continuous subculture tested in this work were shown efficient in the preservation of the 12 isolates during the 4 times 


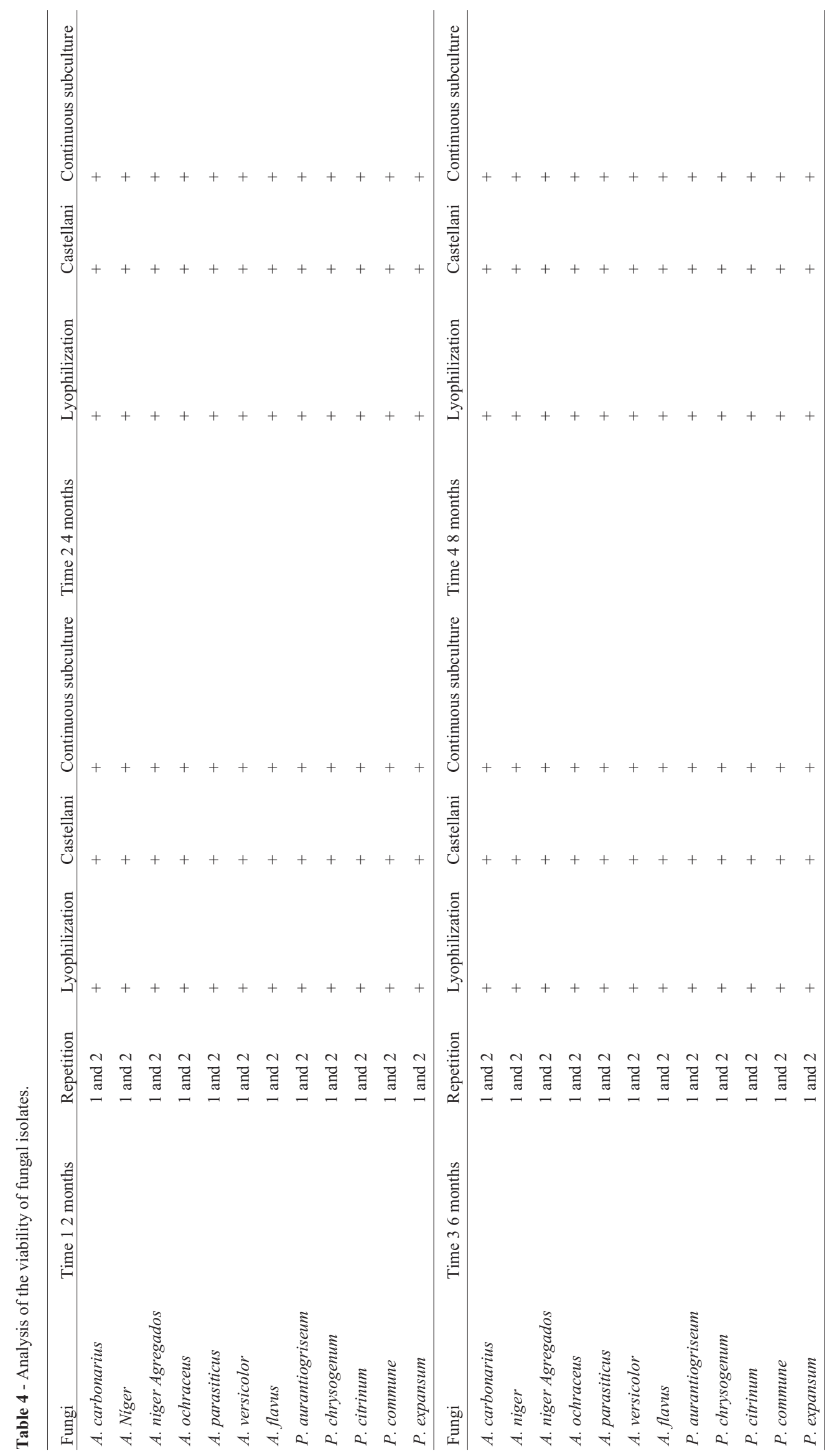


(8 months), however it is important to emphasize that the tested times correspond to a period of short duration, which suggests the need of more lengthy studies.

In relation to the methods used in the present work, the Castellani method is indicated as the most advantageous among the three methods used, therefore besides having a high viability rate and preserving the characteristics of the isolates, it is considered a simple method, one of low cost and that does not need electric power, and thus not being affected by any circumstances due to lack of electricity.

\section{References}

Bueno L, Gallardo R (1998) Preservación de hongos filamentosos en agua destilada estéril. Rev Iberoam Micol 15:166-168.

Cameotra SS (2007) Preservation of microorganisms as deposits for patent application. Biochem Biophys Res Commun 353:849-850.

Christensen CM (1982) The Aspergillus ochraceus group: twe new species from western soils and a synoption. Mycol $74: 210-225$.
Feofilova EP, Kuznetsova LS, Sergeeva YE, Galanina LA (2009) Species composition of food-spoiling mycelial fungi. Microbiol 78:112-116.

Klich MA (2002) Identification of common Aspergillus species. Centraalbureau voor Schimmelcultures, Utrecht.

Nakasone KK, Peterson AW, Jong S (2004) Preservation and distribution of fungal cultures. In: Mueller, G. M.; Bills, G. F.; Foster, M. S. Biodiversity of fungi, inventory and monitoring methods. Elsevier, San Diego, p. 37-47.

Pitt JI (1988) A laboratory guide to common Penicillium species. 2. ed. CSIRO Food, Australia.

Qiangqiang Z, Jiajun W, LI L (1998) Storage of fungi using sterile distilled water orlyophilization: comparison after 12 years. Mycoses 41:255-257.

Romeiro RS (1996) Preservação de culturas de bactérias fitopatogênicas. Universidade Federal de Viçosa, Viçosa, MG.

Samson RA, Houbraken AMP, Kuijpers AFA, Frank JM, Frisvad JC (2004) New ochratoxin A or sclerotium producing species in Aspergillus section Nigri. Stud Mycol 50:23-43.

All the content of the journal, except where otherwise noted, is licensed under a Creative Commons License CC BY-NC. 